\title{
Painful Hemorrhagic Erosions
}

Brian S. Hoyt, MD; Molly C. E. Cowdrey, MD; Marshall A. Guill, MD; Konstantinos D. Linos, MD
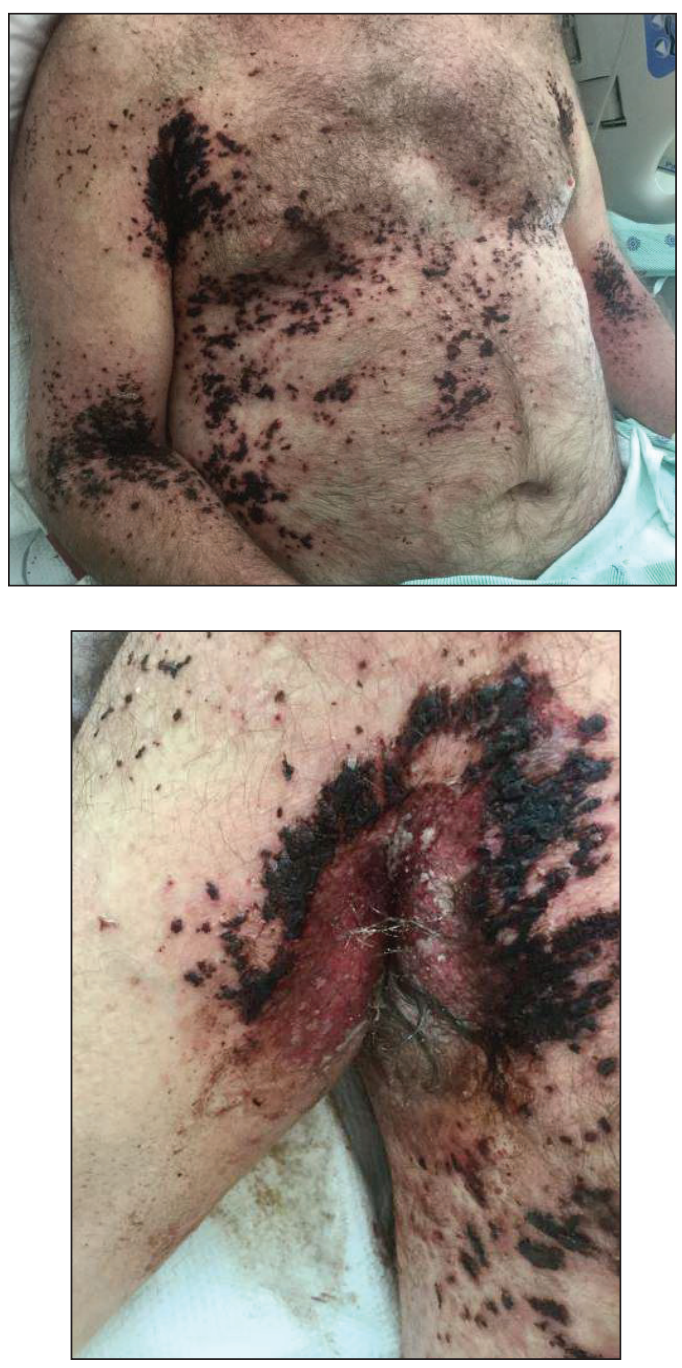

A 62-year-old man with a long-standing history (>40 years) of Hailey-Hailey disease was admitted from an outside hospital due to anemia (hemoglobin, $8.6 \mathrm{~g} / \mathrm{dL}$ [reference range, $14.0-17.5 \mathrm{~g} / \mathrm{dL}])$, thrombocytopenia (platelets, $7 \times 10^{3} / \mu \mathrm{L}$ [reference range, $\left.\left.150-350 \times 10^{3} / \mu \mathrm{L}\right]\right)$, and worsening skin rash. The patient reported that his Hailey-Hailey disease worsened abruptly 1 month prior to admission and had progressed steadily since then. He described the rash as painful, especially with movement. Over the preceding month, he had been treated with topical triamcinolone, topical diphenhydramine, oral prednisone, fluconazole, and oral clindamycin, all without improvement. The skin lesions continued to worsen and persistently bled; he then presented to our institution for further care.

Physical examination demonstrated widespread shallow erosions with hemorrhagic drainage and crusting located on the lower back, chest, abdomen (top), axillae (bottom), groin, arms, and legs. No vesicles or pustules were noted. The patient had no cognitive dysfunction or focal neurologic deficits. A punch biopsy was performed.

\section{WHAT'S THE DIAGNOSIS?}
a. Hailey-Hailey disease flare
b. impetigo
c. Kaposi varicelliform eruption (eczema herpeticum)
d. multiple myeloma
e. tinea corporis

\footnotetext{
From Dartmouth-Hitchcock Medical Center, Lebanon, New Hampshire. Drs. Hoyt, Cowdrey, and Guill are from the Section of Dermatology. Dr. Hoyt also is from and Dr. Linos is from the Department of Pathology and Laboratory Medicine, Dermatopathology Division.

The authors report no conflict of interest.

Correspondence: Konstantinos D. Linos, MD, Division of Dermatopathology, Department of Pathology and Laboratory Medicine, One Medical Center Dr, Lebanon, NH 03766 (Konstantinos.Linos@hitchcock.org).
} 


\section{THE DIAGNOSIS: Kaposi Varicelliform Eruption (Eczema Herpeticum)}

D lymerase chain reaction confirmed presence of herpes simplex virus (HSV) type 1, and the patient was started on intravenous acyclovir $(10 \mathrm{mg} / \mathrm{kg}$ every 8 hours). Diagnosis was further supported by histopathologic examination with confirmatory immunohistochemistry (Figure 1). The patient's anemia and thrombocytopenia also were attributed to widespread HSV infection.

Approximately 8 hours after the patient was started on acyclovir, he developed increasing tremors, confusion, and impaired speech. Lumbar puncture confirmed the presence of HSV-1 in the cerebrospinal fluid. Despite ongoing intravenous antiviral therapy, he required intubation 6 days after hospitalization due to impaired mental status and myoclonic jerking. He remained intubated, unresponsive, and in critical condition for 9 days before he gradually began to demonstrate cognitive recovery. He subsequently was weaned off the ventilator, his
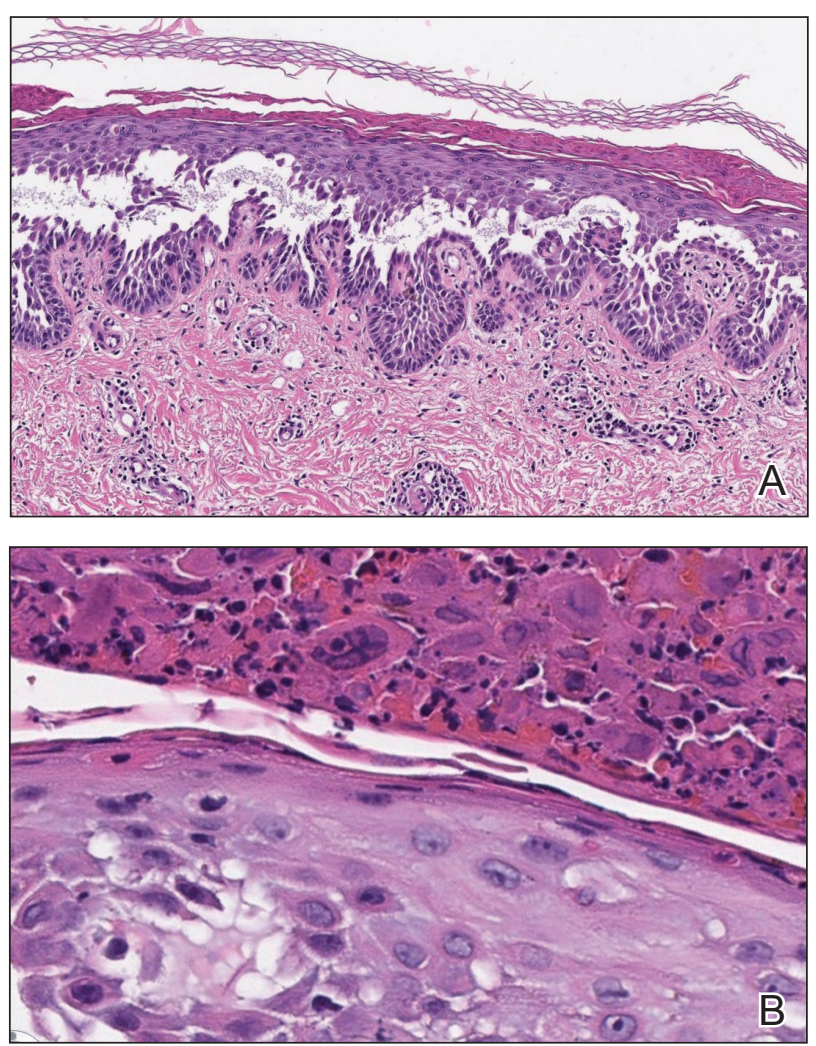

FIGURE 1. A, Background Hailey-Hailey disease with extensive intra-epidermal acantholysis (H\&E, original magnification $\times 100)$. $B$, Superimposed herpes simplex virus infection (eczema herpeticum) demonstrating multinucleated keratinocytes with nuclear molding (H\&E, original magnification $\times 400)$. mental status returned to normal, and his skin rash slowly resolved (Figure 2).

Hailey-Hailey disease (HHD), also known as familial benign chronic pemphigus, is a rare autosomal-dominant condition first described by Howard and Hugh Hailey in $1939 .{ }^{1}$ It is a chronic blistering process characterized by epidermal fragility, often manifesting as macerated fissured erosions in areas exposed to heat and friction (eg, axillae, groin). Hailey-Hailey disease results from a defective calcium transporter (ATP2C1 gene), leading to impaired keratinocyte adhesion. ${ }^{2}$

Eczema herpeticum refers to the dissemination of herpes infection to areas of compromised skin barrier. Although originally used to describe HSV infection in patients with atopic dermatitis, eczema herpeticum has been described in various conditions that affect the skin barrier function, including Darier disease, ichthyosis vulgaris, pemphigus foliaceus, pemphigus vulgaris, and mycosis fungoides, among others. ${ }^{3}$ When applied to skin conditions other than atopic dermatitis, it sometimes is referred to as Kaposi varicelliform eruption. ${ }^{2}$

Hailey-Hailey disease commonly is complicated by a bacterial or fungal infection, including impetigo, tinea, or candidiasis. The first case of HHD complicated by HSV infection was reported in $1973 .{ }^{4}$ A PubMed search of articles indexed for MEDLINE using the terms benign familial pemphigus AND herpes, Hailey-Hailey AND herpes, Hailey-Hailey AND eczema herpeticum, HaileyHailey AND Kaposi varicelliform eruption, and HaileyHailey herpeticum revealed 15 cases of HHD complicated by eczema herpeticum. ${ }^{4-6}$ Herpes simplex virus encephalitis is a rare and life-threatening complication of eczema herpeticum. ${ }^{7,8}$ We report a case of HSV encephalitis resulting from eczema herpeticum in a patient with HHD.

The clinical differential includes a flare of the patient's known HHD, secondary bacterial or fungal infection, or a

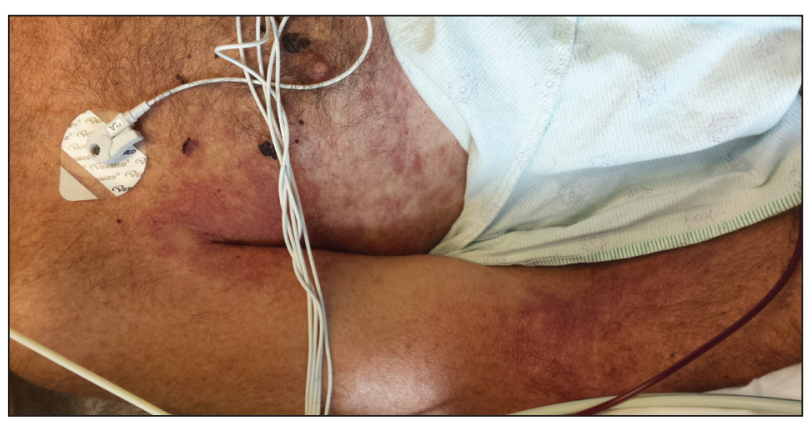

FIGURE 2. Near-complete resolution of erosions and hemorrhagic crusting after treatment with acyclovir. 
PHOTO CHALLENGE DISCUSSION

superimposed viral infection (eg, HSV, zoster). Histologic evidence of herpetic infection would be absent in an uncomplicated flare of HHD. Impetigo is a superficial basterial infection that can present in 2 clinical forms: a vesiculopustular type and less commonly a bulbous type. It is caused by Staphylococcus aureus in most cases. In multiple myeloma with cutaneous dissemination, a monoclonal proliferation of plasma cells would be evident. Lastly, tinea corporis is caused by dermatophyte that can be seen on hematoxylin and eosin or periodic acid-Schiff staining.

The diagnosis of eczema herpeticum in a patient with HHD should be considered in patients who present with grouped vesicles or hemorrhagic or punched-out aerosion in areas of preexisting HHD. The diagnosis can be confirmed by Tzanck smear, viral culture, polymerase chain reaction, or histopathology (with or without amunohistochemistry). . $^{1,26}$ When eczema herpeticum is uspetted, prompt antiviral administration is imperative to limit life-threatening systemic spread.
REFERENCES

1. Hailey J, Hailey H. Familial benign chronic pemphigus. Arch Dermatol. 1939;39:679-685.

2. de Aquino Paul Filho T, deFreitas YK, da Nóbrega MT, et al. Hailey-Hailey disease associated with herpetic eczema-the value of the Tzanck smear test. Dermatol Pract Concept. 2014;4:29-31.

3. Flint ID, Spencer DM, Welkin JK. Eczema herpeticum in association with familial benign chronic pemphigus. J Am Aced Dermatol. 1993;28 (2, pt 1):257-259.

4. Leppard B, Delaney TJ, Sanderson KV. Chronic benign familial pemphigus. induction of lesions by Herpesvirus hominis. Br J Dermatol. 1973;88:609-613.

5. Lee GH, Kim YM, Lee SY, et al. A case of eczema herpeticum with HaileyHailey disease. Ann Dermatol. 2009;21:311-314.

6. Zamperetti M, Pichler M, Perino F, et al. Fin fall won morbus Hailey-Hailey in verbindung mit einem eczema herpeticatum. J Dtsch Dermatol Ges. 2016;14:1035-1038.

7. Ingrand D, Briquet I, Babinet JM, et al. Eczema herpeticum of the child. an unusual manifestation of herpes simplex virus infection. Chin Pediatr (Phila). 1985;24:660-663.

8. Finlow C, Thomas J. Disseminated herpes simplex virus: a case of eczema herpeticum causing viral encephalitis. J R Coll Physicians Edinb. 2018;48:36-39.

E14 I CUTIS ${ }^{\circledR}$

WWW.MDEDGE.COM/DERMATOLOGY

Copyright Cutis 2020. No part of this publication may be reproduced, stored, or transmitted without the prior written permission of the Publisher. 